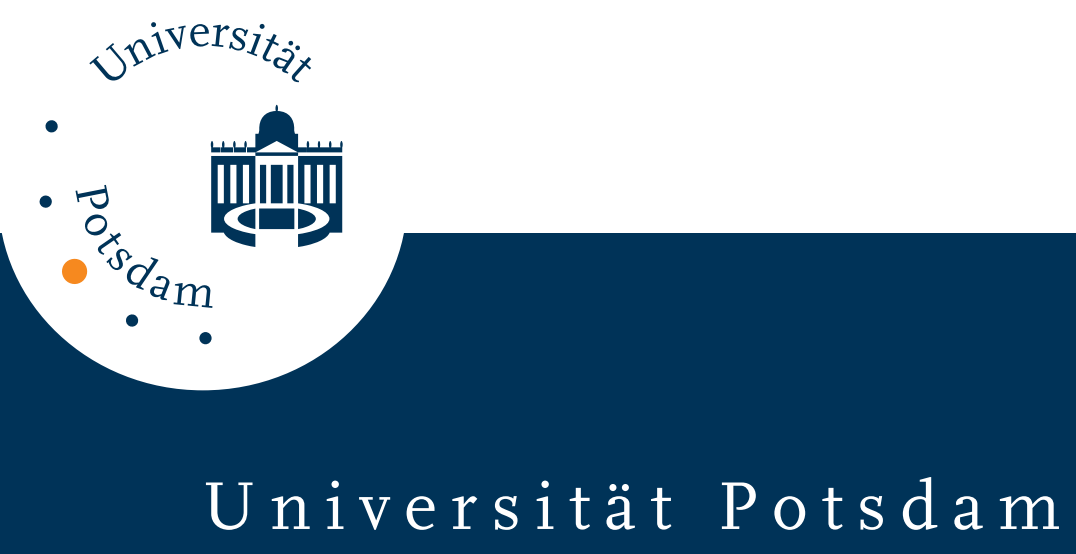

Matthias Bluemke, Ralf Brand, Geoffrey Schweizer, Daniela Kahlert

\title{
Exercise might be good for me, but I dont feel good about it : Do automatic associations predict exercise behavior?
}

first published in:

Journal of sport and exercise psychology 32 (2010), 2, S. 137 - 153

Postprint published at the Institutional Repository of the Potsdam University:

In: Postprints der Universität Potsdam

Humanwissenschaftliche Reihe ; 178

http://opus.kobv.de/ubp/volltexte/2010/4251/

http://nbn-resolving.de/urn:nbn:de:kobv:517-opus-42510

Postprints der Universität Potsdam

Humanwissenschaftliche Reihe ; 178 



\title{
Exercise Might Be Good for Me, But I Don't Feel Good About It: Do Automatic Associations Predict Exercise Behavior?
}

\author{
Matthias Bluemke, ${ }^{1}$ Ralf Brand, ${ }^{2}$ Geoffrey Schweizer, ${ }^{2}$ \\ and Daniela Kahlert ${ }^{2}$ \\ ${ }^{1}$ University of Heidelberg; ${ }^{2}$ University of Potsdam
}

\begin{abstract}
Models employed in exercise psychology highlight the role of reflective processes for explaining behavior change. However, as discussed in social cognition literature, information-processing models also consider automatic processes (dualprocess models). To examine the relevance of automatic processing in exercise psychology, we used a priming task to assess the automatic evaluations of exercise stimuli in physically active sport and exercise majors $(n=32)$, physically active nonsport majors $(n=31)$, and inactive students $(n=31)$. Results showed that physically active students responded faster to positive words after exercise primes, whereas inactive students responded more rapidly to negative words. Priming task reaction times were successfully used to predict reported amounts of exercise in an ordinal regression model. Findings were obtained only with experiential items reflecting negative and positive consequences of exercise. The results illustrate the potential importance of dual-process models in exercise psychology.
\end{abstract}

Keywords: exercise, health behavior, automatic processes, evaluative priming, affective priming

Sufficient levels of activity are health enhancing (Kesaniemi et al., 2001). Yet, less than half (49\%) of the U.S. population (Haskell et al., 2007) and little less than one-third (31\%) of the people living in European Union countries (Sjöström, Oja, Hagströmer, Smith, \& Bauman, 2006) reach proposed levels. To prevent health problems such as heart failure, diabetes mellitus, hypertension, or specific types of cancer, the following amounts of activity are recommended (Haskell et al., 2007): $30 \mathrm{~min}$ of moderate-intensity aerobic physical activity (e.g., brisk walking) on 5 days every week, or 20 min of vigorous-intensity aerobic physical exercise (e.g., inline-skating or running) 3 times a week.

Bluemke is with the Department of Social Psychology, University of Heidelberg, Heidelberg, Germany. Brand, Schweizer, and Kahlert are with the Department of Sport and Exercise Psychology, University of Potsdam, Potsdam, Germany. 


\section{Behavior Change Models}

Changing habitual physical inactivity is evidently a difficult task (Baranowski, Anderson, \& Carmack, 1998; Baranowski, Lin, Wetter, Resnicow, \& Hearn, 1997; King, 2001; Sallis, 2001). One recent meta-analysis provides a particularly informative summary of the effectiveness of up-to-date health behavior interventions (Webb \& Sheeran, 2006). It is exclusively based on studies with experimentally manipulated intentions. Different models and interventional approaches were investigated. Its most important result is that health behavior interventions based on psychological models of behavior change are often pretty good at altering intentions $(d=0.66,95 \% \mathrm{CI}=.51$ to .82$)$. But they are worse at altering behaviors $(d=0.36$, $95 \% \mathrm{CI}=.22$ to .50$)$. There are also meta-analytic results calculated exclusively for the physical activity domain (Hagger, Chatzisarantis, \& Biddle, 2002). According to these results, the theory of planned behavior (TPB; Ajzen, 1991), for example, is able to account for $45 \%$ of variance in activity intentions, compared with only $27 \%$ of the variance in activity behavior. Although these results confirm that TPB is capable of explaining a fair share of (planned) physical activity (or exercise, or other behaviors), a substantial portion of behavioral variance remains unexplained.

The problem of unexplained behavioral variance is sometimes referred to as the intention-behavior-gap (Sheeran, 2002). One way to deal with it emphasizes the role of volitional processes (Schwarzer, 2008). The differentiation between motivational and volitional aspects of behavior change is evident, for example, in the health action process approach (HAPA; Schwarzer, 1992), which has been repeatedly applied to the domain of exercise (e.g., Lippke, Ziegelmann, \& Schwarzer, 2004; Scholz, Sniehotta, \& Schwarzer, 2005). The results are encouraging, although meta-analyses that could give an impression about probable improvements in explained variance are missing. A second way to deal with it is to address the role of affect, and, for example, to explore the affective component of attitudes (for the distinction between cognitive and affective components of attitudes, see Zanna \& Rempel, 1988). According to this line of thought, people do not only exercise because they know (cognitive component) that exercising is good for their health, but also because they feel (affective component) good about exercising. In a study on exercise participation (Brand, 2006), cognitive and affective attitudinal components were included separately in a single binary logistic regression model. Exercise participation served as the dependent variable. Only the affective $(\beta=.57$, $p<.01)$ and not the cognitive component $(\beta=.24, n s)$ contributed significantly to the model. A growing number of studies contribute to this area of affect-related research. Some directly corroborate reported findings (Kiviniemi, Voss-Humke, \& Seifert, 2007); others point to the importance of appropriate emotional appraisals (McAuley, Jerome, Elavsky, Marquez, \& Ramsey, 2003; Mohiyeddini, Pauli, \& Bauer, 2009).

\section{Social Information Processing Models}

Social cognition research has taken a different approach in its endeavor to link cognition with action. Following such influential models on the attitude-to-behavior relation-for example, Fazio's (1990) motivation and opportunity as determinants (MODE) model - so-called dual-process models distinguish a deliberative or reflective mode from a heuristic or intuitive mode of information processing (Chaiken 
\& Trope, 1999; Smith \& DeCoster, 2000; Evans, 2008). From this point of view, attitudes can determine behavior either by means of reflective, explicit processing (resembling the attitude-behavior-relationship depicted in TPB, e.g.), or via an intuitive rather unconscious route. Central features of different dual-process models are combined in the reflective-impulsive model (RIM) proposed by Strack and Deutsch (2004).

Although these routes were conceived of as independent and distinct, RIM posits the parallel processing of information in two separate systems. Nevertheless, they are connected via several pathways, which allow for mutual influences. According to RIM, only the reflective system weighs knowledge, for example about personal values or probabilities of behavioral consequences. As a result of this relatively slow process, a behavioral option is chosen, before an intention for the (planned) behavior is formed. Behavioral schemata are then further ignited and carried out by these intentions. The impulsive system, on the other hand, allows for parallel processing in associative semantic networks. The basic idea is that mere perception of stimuli (and in some situations even complex reflective processes themselves) may activate further elements of the network in the impulsive system. Behavioral schemata are initiated via a nonreflective, automatic route. Associative links between network elements (concepts) are considered to be the result of learning episodes and may be relatively hard to overcome.

For instance, environmental characteristics that frequently co-occur with affective reactions will result in conditioning effects that may not be represented in the reflective system but, nevertheless, exist on the automatic level. People who have experienced exercise as being unpleasant would associate exercise with negative valence (or vice versa). Once a valence-laden association is established within the impulsive system, this association may influence the probability that the behavior is executed. Positive associations will increase a person's inclination to show the behavior. Negative ones will decrease it.

As a cautionary note, the existence of two separate social-cognitive systems remains much debated. On the one hand there is neuroscientific evidence that points to a biological separation of two systems (Lieberman, 2007). On the other hand evidence suggests that proposing two distinct systems might be too simple (Evans, 2008). Several inconsistencies in researchers' descriptions of systems provide support for this notion. For example, there are different terminologies used to name the accompanying processes as well as differences in the main features attributed to the systems. Future research will show whether distinguishing type I and type II processes will be more informative than continuing to refer to processes located at System 1 and System 2. Type II processes would require access to a working memory source of limited capacity, whereas type I processes would not (Evans, 2008). Adopting this terminology, automatic processes, so far attributed to the impulsive system, would represent a rather heterogeneous group of type 2 processes.

\section{Explorations on the Impulse to Exercise}

But why should exercise psychologists care about associative links in the impulsive network at all? Hofmann, Friese, and Wiers (2009) showed that impulsive and reflective processes are connected differentially to at least some health-related behaviors, for example eating and drinking, drug abuse, or sexual interest: When 
people have the opportunity and are motivated to deliberate, the reflective system will govern their behavior. In contrast, when motivation is low and time or capacity for deliberation is sparse, the impulsive system will become more important (Friese, Hofmann, \& Schmitt, 2008; Friese, Hofmann, \& Wänke, 2008). Therefore, according to circumstances, the two systems may give rise to conflicting behavioral tendencies (Figure 1). On the one hand explicit deliberation may lead to an intention to engage in exercising. This scenario can be modeled and straightforwardly explained with TPB or HAPA. On the other hand, exercise might be associated with negative valence at the automatic level. Taking both systems and their potential influence on behavior into account, implicit associations on the automatic level could be used to understand activity or inactivity behavior in an additional class of situations, in which there is no time or space for reflecting plans or intentions. Exploring the impulsive system could therefore lead to improve predictions of behavior.

As a necessary step toward investigating the impulsive system in the domain of exercise behavior, we present an empirical study to demonstrate that such automatic processes are as relevant in this domain as in other domains (see Strack \& Deutsch, 2004, for an overview; Hofmann et al., 2009, for other health-related domains). With the present article, we focus on automatic evaluative associations to arise with representations of exercise behavior. The central proposal is that interpersonal differences in exercise behavior are correlated with different evaluative associations, formed and stored in the impulsive system: Exercisers might automatically associate exercising with positive evaluations. Inactive people might associate the

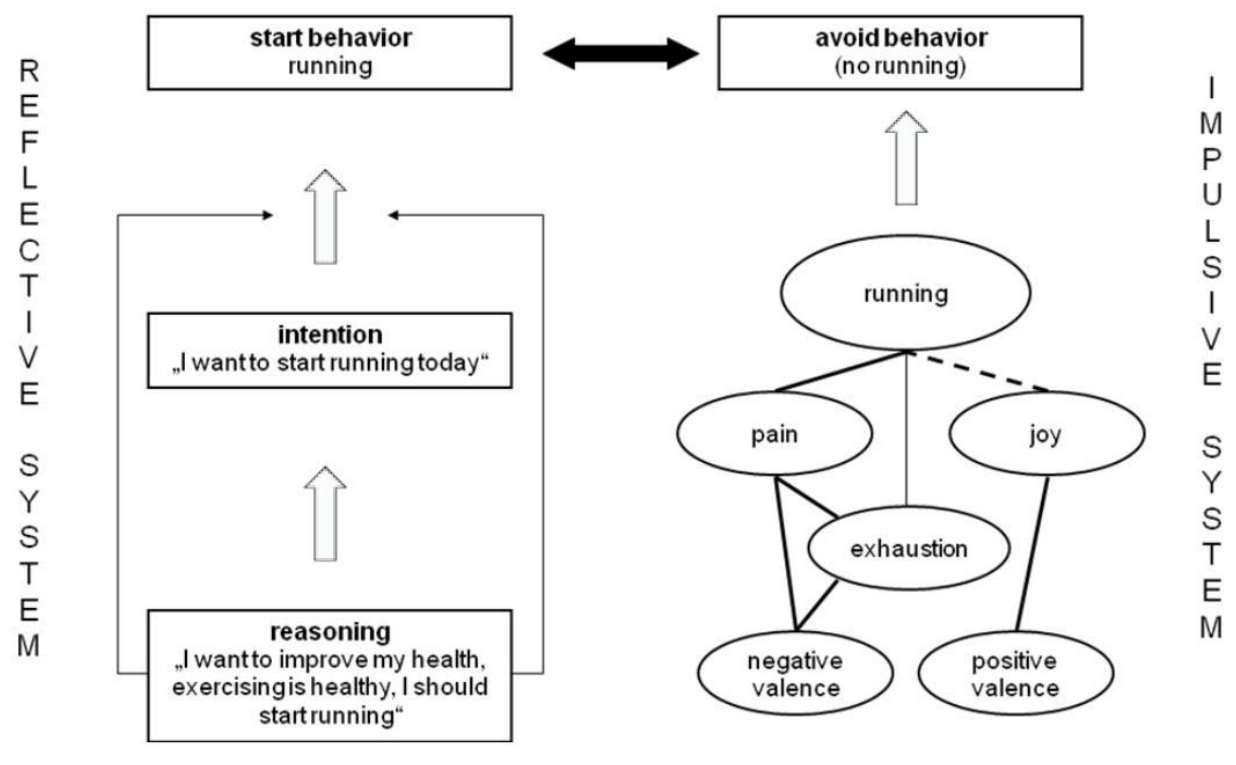

Figure 1 - Illustration of competing behavioral tendencies: While the re囚ective system tends to activate behavior (due to positive outcome expectancies), the impulsive system tends to avoid the same behavior (due to strong and stable associations with negatively evaluated experiences). 
idea of exercising — spontaneous and rather unconsciously_with less positive or even negative evaluations.

To assess associative structures within the impulsive system, reaction-timebased, indirect measures have been firmly established (Fazio \& Olson, 2003). One of them is the evaluative priming task (Fazio, Sanbonmatsu, Powell, \& Kardes, 1986). For drawing inferences about automatic associations in the impulsive system, reaction times of a few hundred milliseconds are measured while subjects are working on computer-based sorting tasks. Participants do not have to form deliberate answers, which might be biased by socially desirable responding (Degner, Wentura, \& Rothermund, 2006).

With the current study, we test the hypothesis that groups of students who engage in regular physical exercise have more favorable automatic associations on exercise than students who do not regularly exercise. In addition this priming effect (i.e., measured reaction time differences) shall be tested in its ability to predict the amount of participants' self-reported exercise behavior.

\section{Method}

\section{Sample}

Ninety-four participants, 47 female and 47 male, were recruited at the Universities of Heidelberg and Stuttgart in exchange for a compensation of $5 €$. Their mean age was 23 years $( \pm 3.3$ years $)$.

\section{Instruments}

Exercise Questionnaire. To distinguish participants who exercise or not, we used a short and simple scoring algorithm based on the transtheoretical model (TTM; Prochaska \& DiClemente, 1983). This algorithm allows for classifying exercisers vs. nonexercisers as belonging to one of five different stages (precontemplation, contemplation, preparation, action, or maintenance) of behavior change. The classification is based on participants' answers to the question, "Do you regularly (i.e. more than two times a month) exercise (e.g. walking, swimming, gymnastics, tennis or the like)?" Categories range from 1 to $5(1=$ no, and not intending to start within the next 6 months; $2=$ no, but intending to start within the next 6 months; $3=$ no, but intending to start within the next 30 days; $4=$ yes, for less than 6 months; $5=$ yes, for more than 6 months). Times used to define category boundaries resemble conventions as recommended in original TTM work (Prochaska et al., 1994). The algorithm's criterion for regular exercising was set to "more than two times a month." This criterion is different from established health-enhancing physical activity (HEPA) guidelines (the HEPA recommendation, as paraphrased in the first paragraph of this paper) and different from the one used in the original TTM algorithm (3-5 times per week for 20-60 min per session). The idea behind this adaptation was that occasionally exercising subjects (e.g., those who manage to exercise from time to time, but not regularly according to HEPA) should rather be sorted into the exercising group than into the inactive group. Reliability and validity of the stages of change algorithm are disputed every once a while. In many studies, the criteria and times used to define category boundaries are chosen 
somewhat arbitrarily (Marshall \& Biddle, 2001). In our study, the TTM algorithm was used for simple screening purposes to reach the intended numbers of regularly exercising and nonexercising subjects. Controversial psychometrical properties did not constitute a problem for our purposes therefore.

The frequency and duration of participants' engagement in exercise was assessed more precisely using two self-constructed ordinal scales. Participants indicated the frequency of exercises using a 5 -point scale $(1=$ less than once a week; 2 = once $a$ week; 3 = two times a week; $4=$ three times a week; $5=$ more than three times a week). Subsequently, subjects reported average duration of exercise units using a 3 -point scale $(1=$ less than $20 \mathrm{~min} ; 2=$ between 20 and $40 \mathrm{~min} ; 3=$ more than $40 \mathrm{~min}$ ). We used the weekly question format, as we considered the relevant information to be better accessible when assessed per week instead of per month (Schwarz \& Oyserman, 2001).

Priming Procedure. The evaluative priming method has proven useful for assessing automatic evaluations and predicting related behaviors (Eves, Scott, Hoppe, \& French, 2007; Fazio, Jackson, Dunton, \& Williams, 1995; Fazio et al., 1986). Participants are instructed to solve a task with two features: While working on a series of prime-target combinations at the computer, participants have to neglect the presented positive or negative prime stimulus (first feature of the priming procedure), before the valence of a following positive or negative target stimulus has to be correctly identified as fast as possible (second feature of the priming procedure). Targets remain on the screen until the participant indicates its perceived valence by pressing one of two response keys (for positive and negative valence respectively). A cross, presented for $1000 \mathrm{~ms}$ at the center of the screen, indicates the beginning of each trial and focuses the participant's attention on the following prime presentation. Each trial ends with a blank screen lasting $2000 \mathrm{~ms}$ (Figure 2).

The notion of an evaluative priming effect refers to the automatic activation of valence after the supra- or subliminally encountering of a prime stimulus. The elicited valence can subsequently lead to either response facilitation, when target stimuli are subjectively classified as valence congruent with previous prime presentations, or response inhibition in valence- incongruent trials. Primes tend to activate either the associated positive or negative valence within a few hundred milliseconds. This priming effect is often interpreted in terms of spreading activation within a participant's semantic network and can be used to diagnostically infer the evaluation of primes (Fazio \& Olson, 2003). If conceptually similar prime stimuli elicit specific valence across many trials, the evaluation of the prime concept can be calculated from the mean response latencies of the various prime-target combinations, or, more specifically, by the amount of facilitation and inhibition on congruent and incongruent target trials (see Figure 2).

For instance, a positive priming effect of $30 \mathrm{~ms}$ (indicating positive associations toward exercising) may result when a participant is relatively slow to categorize negative target words following exercise primes $\left(\mathrm{RT}_{\text {Negative }}\right.$ arget Exercise Prime $=840$ $\mathrm{ms}$ ), while controlling for the latency of negative target words after control primes $\left(\mathrm{RT}_{\text {Negative Target } \mid \text { Control Prime }}=830 \mathrm{~ms}\right)$; at the same time, the participant is relatively fast to categorize positive target words following exercise primes ( $\mathrm{RT}_{\mathrm{P}}$ $=730 \mathrm{~ms}$ ), while controlling for the late primes ( $\mathrm{RT}=750 \mathrm{~ms})$. The priming effect is then calculated as follows: $(840 \mathrm{~ms}-830 \mathrm{~ms})-(730 \mathrm{~ms}-750 \mathrm{~ms})=10 \mathrm{~ms}-(-20 \mathrm{~ms})=30 \mathrm{~ms}$. 


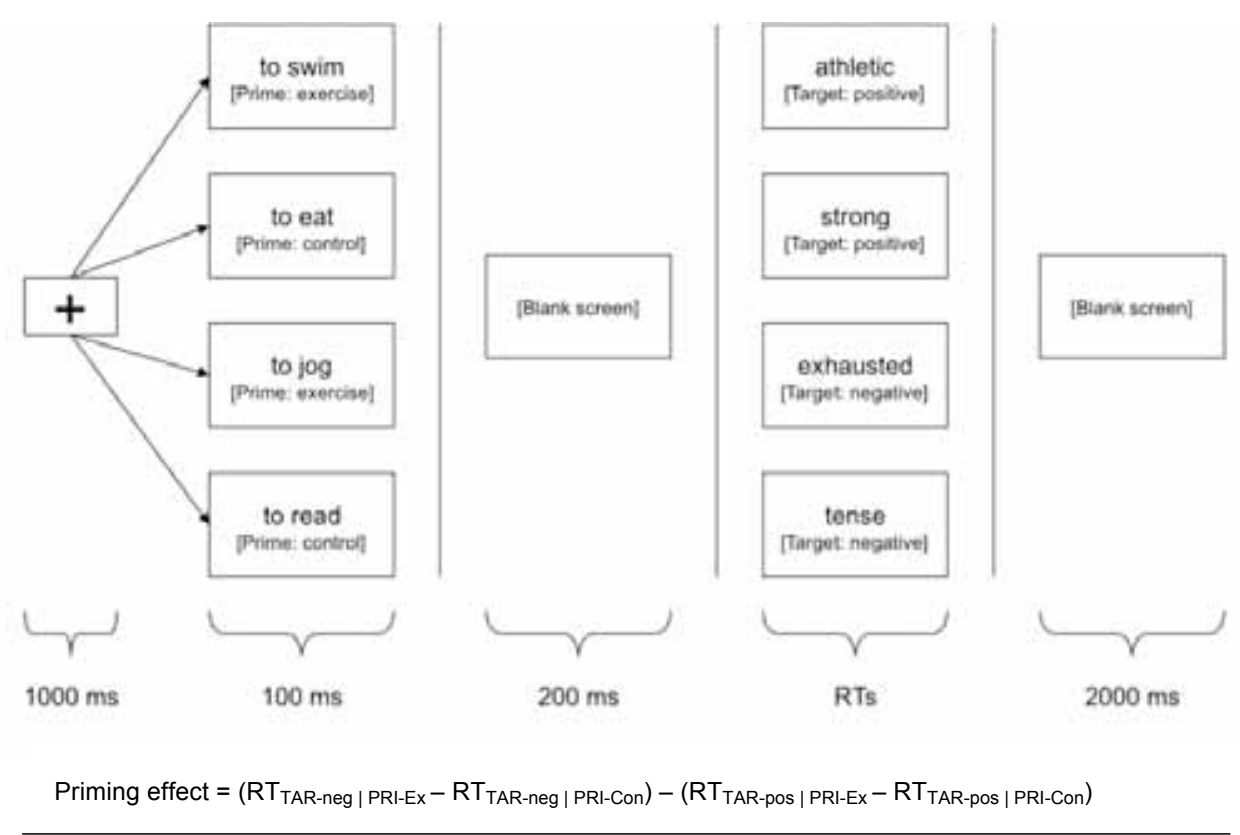

Figure 2 - Priming procedure and calculation of priming effect, exemplified for the exercisespecific target stimuli (German verbs do not need particles: The "to" in prime words is added here as a consequence of the English translation; $\mathrm{RT}=$ mean reaction time; $\mathrm{PRI}=$ prime; $\mathrm{TAR}=$ target; $\mathrm{Ex}=$ exercise Con = control; $\mathrm{Pos}=$ positive; $\mathrm{Neg}=$ negative $)$.

Our participants worked on two priming variants: half of the positive and negative target stimuli were adjectives, semantically related to outcomes of exercising ("exercise-specific"; e.g., athletic vs. exhausted). The other half comprised exercise-unspecific positive or negative adjectives ("generic"; e.g., patient vs. corrupt). We included this variation of positive and negative target stimuli to set up two variants of evaluative priming, allowing for a conceptual replication, combined with an extension of Fazio et al.'s (1995) standard procedure. Not only did we want to measure evaluations in a broad (i.e., generic) sense, but also with exercise-specific concepts. This is because recent empirical findings have pointed to the importance of activating associations context-specifically (e.g., Rydell \& Gawronski, 2009). Automatic evaluations elicited by exercise primes might be tapped exclusively by either generic target words, or exclusively by exercise-specific target words, in a similar fashion by both, or not at all.

\section{Procedure and Design}

Prestudy. Stimuli for the priming procedure were tested in an independent prestudy using the data of a subsample of sports major and nonsports students $(N$ $=16$ ). We pretested a sample of verbs to be used as prime stimuli (first feature of the evaluative priming procedure) and a sample of adjectives to be used as target stimuli (second feature of the priming procedure). To assess each item's explicit 
connotations with exercise, we used a 9-point scale, with anchors labeled weak (1) or strong association with exercise (9). To assess valence, we used another 9-point scale, with anchors labeled negative (1) or positive (9). The concept of exercise was left undefined to allow for natural variation in participants' understanding of the concept.

For the first feature of the priming procedure, 10 verbs were chosen as exercise primes (e.g., [to] swim, [to] jog, [to] exercise; verbs are to be presented without particles in German, the language in which our study was conducted). The same number of exercise-unrelated verbs served as control primes (e.g., [to] eat, [to] read, [to] wash). Exercise and control primes differed significantly with regard to their relation to exercise, $M \mathrm{~s}=7.96(S D=0.73)$ vs. $3.80(S D=1.36), t(18)=$ $8.53, p<.001$, Cohen's $d=3.81$. Exercise and control primes did not differ in word length, $M \mathrm{~s}=7.1(S D=1.51)$ vs. $7.4(S D=1.56), t(18)=0.44, n s, d=0.20$. They did not differ in valence, $M \mathrm{~s}=6.29(S D=1.08)$ vs. $6.39(S D=1.14), t(18)$ $=-0.20, n s, d=-0.09$.

For the categorization task of positive and negative stimuli (second feature of the priming procedure) we used 20 positive and 20 negative adjectives as target stimuli. Positive and negative words were equal in word length, $M \mathrm{~s}=6.95$ ( $S D=$ $2.11)$ vs. $7.15(S D=2.10), t(38)=-0.30, n s, d=-0.09$. Positive and negative targets differed clearly in valence, $M \mathrm{~s}=8.14(S D=0.47)$ vs. $1.91(S D=0.56), t(38)$ $=38.32, p<.001, d=12.12$. Within this pool, the target words represented two different sets: either a subset of 10 generically positive and 10 generically negative adjectives (e.g., patient vs. corrupt), or a subset of 10 positive and 10 negative adjectives semantically related to possible consequences of exercise (e.g., athletic vs. exhausted). Generic and exercise-related stimulus sets proved to be differently connoted with exercise, $M \mathrm{~s}=3.58(S D=1.03)$ vs. $6.24(S D=1.84), t(38)=-5.65$, $p<.001, d=-1.76$. Words did not differ with regard to valence, $M \mathrm{~s}=4.92(S D=$ $3.35)$ vs. $5.13(S D=2.96), t(38)=-0.21, n s, d=-0.07$.

Main Study. Recruiters approached potential subjects at appropriate places on campus. All the persons who were addressed were briefly screened using the items of the TTM scoring algorithm. As indicated by their answers to these items, they were assigned either to the nonexercisers group (TTM-stage 1 or 2) or to one of the two regular-exercisers groups (TTM-stage 5; see below). People who told the recruiters they belonged either to TTM-stage 3 or to TTM-stage 4 were not subjected to further investigation. Recruitment of participants stopped once the sufficient number of subjects ( $>30$ per group) was reached.

The first group consisted of university students majoring in sports and exercise science. All of them engaged in exercise more than two times a month, for more than the last six months (TTM-stage $5 ; n_{1}=32$ ). We expected sport students to have a rather unique perspective on sport and exercise due to their academic knowledge or specific personal experiences that had led them to major in this subject. Therefore we decided to collect a second group of regularly exercising students. None of these students were sport students, but all of them engaged in physical exercise more than two times a month for more than the last 6 months (TTM-stage $5 ; n_{2}=$ 31). The third group encompassed students who reported to be physically inactive (in the sense that they did not engage more than twice a month in sport or exercise activities), and who were not willing to become more active within the next month (TTM-stage $1-2 ; n_{3}=31$ ). 
After having obtained participants' informed consent, the experimenter measured automatic evaluations of exercise as indicated by the priming procedure. We were interested in the spontaneous evaluations of exercise primes, which occurred after having presented these primes supraliminally for $100 \mathrm{~ms}$ (Figure 2). Each participant received two priming tasks. Each priming variant contained identical exercise and control primes. However, priming variants differed with regard to target stimuli: one variant applied the set of exercise-specific positive and negative target stimuli (e.g., athletic vs. exhausted), whereas the other variant was made up by the set of generically positive and negative target stimuli (e.g., patient vs. corrupt). Both priming variants were presented in counterbalanced order across the sample. Within each of the priming variants, participants worked on 120 randomly drawn prime-target combinations that followed an orthogonal 2 (prime content: exercise vs. nonexercise) $\times 2$ (target valence: positive vs. negative) design with repeated measurement on both factors. Priming variants took approximately 5-10 min each.

Finally, participants were asked to classify personal frequencies and durations of exercise sessions using the paper-pencil questionnaire explained above. As expected, the three groups (nonexercising students, exercising nonsport students, and sport and exercise majors; all numbers in this paragraph are reported in this order) differed in frequency of physical exercises per week. According to a Kruskal-Wallis test of rank orders, the frequency mean ranks amounted to 15,46 , and $64, \chi^{2}(2, N=87)=54.61, p<.01$. The average frequency categories chosen were $1.29,3.58$, and 4.53. This translates roughly into less than 1 time per week, almost 3 times a week, or even more often. In addition, the duration of typical exercise sessions differed according to the categories chosen-on average, 1.91, 2.84, and 2.97 respectively. This indicates that the sessions lasted either less than 40 min or almost always more than $40 \mathrm{~min}$, resulting in significantly different mean ranks $(25,47$, and 52$), \chi^{2}(2, N=85)=31.16, p<.01$. The number of subjects for Kruskal-Wallis tests slightly vary: Some participants did not answer the respective questions pertaining to frequency and length of exercise accurately, after they had initially indicated (according to TTM-categories 1 or 2 ) that they were not active at the time. In sum, the sample displayed a wide range of proclivity to physical exercise. However, there were no other group differences, in terms of age, $F<1$, or gender, and differences were almost equally distributed across the subsamples (with gender proportions ranging between .48 and .52).

Altogether the experimental procedure and questioning lasted about $30 \mathrm{~min}$. Participants were thanked and thoroughly debriefed before they received their reward. The research procedure was approved by the universities of Heidelberg and Stuttgart.

\section{Results}

Inspection of the data shows that participants identified target valence quickly (on average $663 \mathrm{~ms}$ in the generic priming variant and $729 \mathrm{~ms}$ in the exercise-specific priming variant). They made few errors in both priming procedures $(5.2 \%$ and $6.8 \%$ of the trials, respectively). A significant, though theoretically not very enlightening, discrepancy between mean sorting speed of generic and exercise-specific target stimuli is apparent, paired-t $(92)=-5.97, p<.001$, Cohen's $d=-0.44$ (we calculated $d$ by dividing the difference score by the original standard deviation of the sample, 
rendering $d$ comparable in size to a between-group design, rather than basing $d$ on the paired- $t$ test value; Dunlap, Cortina, Vaslow, \& Burke, 1996). The number of errors did not differ significantly, paired- $t(92)=-1.18, p=.24, d=-.15$. To facilitate the interpretation of latency outcomes, we prepared the latency measure in line with common recommendations. Single latency differences per priming task indicate the evaluative priming effect of exercise primes relative to control primes. Two kinds of priming effects were calculated, one raw effect and one ipsatized effect. Raw priming effects are directly expressed in measured milliseconds. The ipsatized priming effect was calculated by subtracting a participant's individual mean latency from each trial latency and dividing by her overall standard deviation. The result of this calculation is that latencies are free from interindividual differences due to participants' mean response levels. This variable is comparable to $z$-standardizing values, but on a subject's individual basis. Raw and ipsatized priming effects were separately subjected to two two-factorial mixed ANOVAs, with group as betweensubject factor and type of priming task as repeated-measurement factor.

A look at the priming effects based on raw latencies confirmed that, while there were no mean differences between the two types of priming, $F(1,90)=0.49, n s, \eta^{2}$ $=.01$, there was a tendency toward group differences across both measures, $F(2,90)$ $=2.85, p=.06, \eta^{2}=.06$. Sports students and regularly exercising nonsport students experienced a relative facilitation (in the size of $13 \mathrm{~ms}$ for sport students, $25 \mathrm{~ms}$ for nonsport students) of positive words after exercise primes, relative to negative words after exercise primes (thus showing more positive than negative associations about exercising). Nonexercising participants were faster in responding to negative words

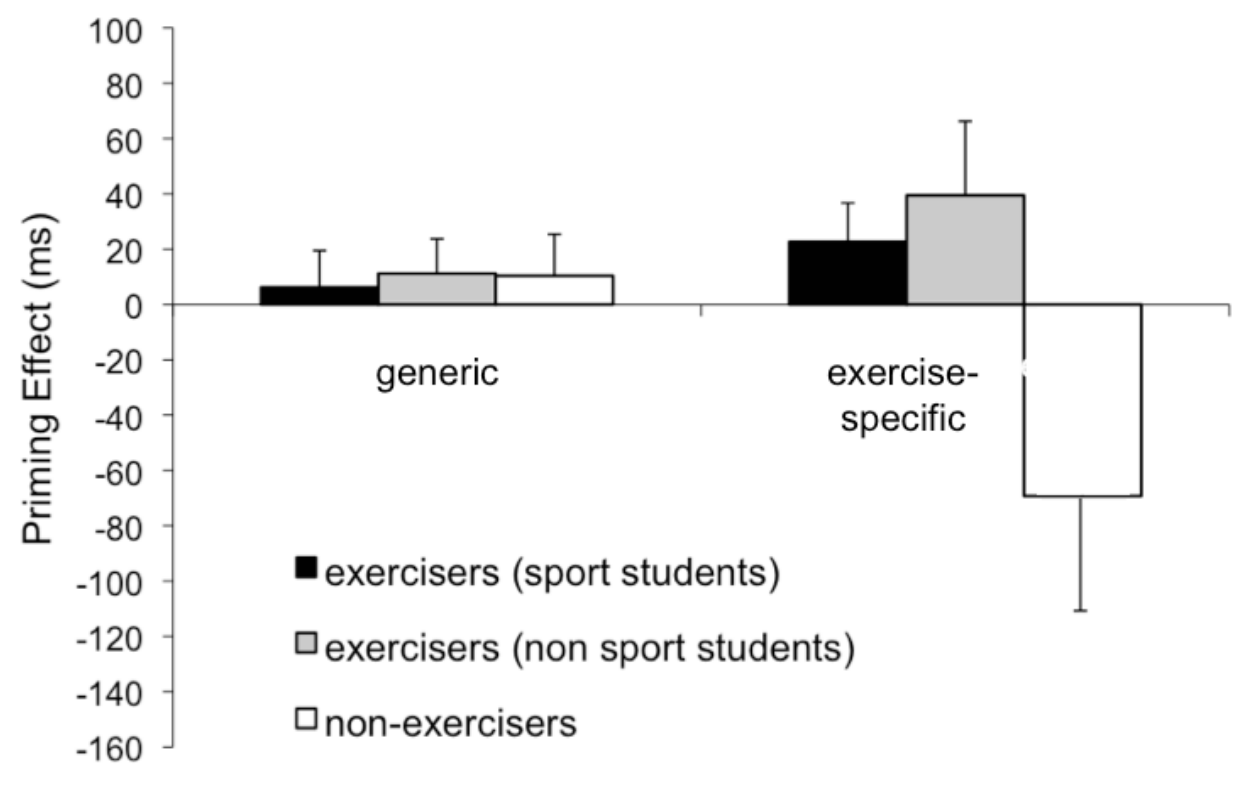

Figure 3 - Mean group differences (priming effects [in milliseconds]) in the evaluative priming tasks split per condition (generic vs. exercise-specific priming variant). Error bars represent standard errors. 
after exercise primes $(-29 \mathrm{~ms})$, indicating more negative associations. Importantly, a significant two-way interaction showed that these tendencies were driven by one type of priming task, $F(2,90)=3.52, p=.03, \eta^{2}=.07$. When splitting the analysis into separate ANOVAs for each type of priming (i.e., the exercise-specific variant and the generic one), group differences were clearly evident in the exercise-specific variant, with $M \mathrm{~s}=23 \mathrm{~ms}(S D=79)$ for exercising sport students, $39 \mathrm{~ms}(S D=150)$ for exercising nonsport students, and $-69 \mathrm{~ms}(S D=231)$ for nonexercising students, $F(2,91)=3.93, p=.02, \eta^{2}=.08$. There were no such differences in the generic variant, with $M \mathrm{~s}=6 \mathrm{~ms}(S D=74)$ for exercising sport students, $11 \mathrm{~ms}(S D=70)$ for exercising nonsport students, and $10 \mathrm{~ms}(S D=84)$ for nonexercising students, $F(2,90)=0.04, n s, \eta^{2}=.01$. Thus, exercise was positively evaluated within a few hundred milliseconds in exercising groups, but only the priming measure relying on exercise-specific targets was capable of demonstrating this relationship (Figure 3).

The analyses of priming effects (i.e., the interaction of priming task $\times$ group) on ipsatized scores confirmed these conclusions, $F(2,90)=3.22, p=.05, \eta^{2}=$ .07 (other $F \mathrm{~s} \leq 1.05, p \mathrm{~s}>.35, \eta^{2} \mathrm{~s}<.02$ ). Comparisons of means showed that the priming effects (units in ipsatized scores) were significantly different in the priming variant with exercise-specific target stimuli, with $M \mathrm{~s}=0.10(S D=0.44)$ for exercising sport students, $0.07(S D=0.46)$ for exercising nonsport students, and -0.18 $(S D=0.52)$ for nonexercising students, $F(2,91)=3.43, p=.04, \eta^{2}=.07$. Again, there were no such differences in the generic item pool with positive and negative target words, with $M \mathrm{~s}=0.01(S D=0.42)$ for exercising sport students, -0.01 ( $S D$ $=0.35)$ for exercising nonsport students, and $0.05(S D=0.41)$ for nonexercising students, $F(2,90)=0.23, n s, \eta^{2}=.01$. Having demonstrated that only one of the priming variants was a sensitive indicator of differences in automatic evaluation, it does not come as a surprise that both types of priming (the exercise-specific variant and the generic one) did not correlate substantially, $r \mathrm{~s}=.11(p=.30)$ and $.12(p=$ $.24)$, for the raw and ipsatized priming effects respectively.

Given that the separation into three groups can be considered arbitrary, and a lot of within-group variance is not accounted for, in addition, we have correlated the priming scores with self-reported frequency, duration, and overall amount of exercises. The amount of overall exercising was calculated by multiplying the category values for frequency with the category values denoting duration. All three criteria (frequency, duration, and their product; coefficients reported in this order) were related to exercise-specific priming effects, as evident in significant rank-order correlations. Results were Spearman's $\rho=.21, .32$, and $.28(p s<.05)$ for raw priming effects, and $\rho=.25, .34$, and .31 ( $p$ s $<.02)$ for ipsatized priming effects respectively. Any trends for the generic priming variant were far from being significant, with $\rho=.10$ at most $(p s>.38)$.

On account of these significant relationships, an ordinal regression approach was used to predict the choice of category on each of the rating scales that indicated self-reported frequency and duration (as well as the combination thereof). The ipsatized exercise-specific priming effect was chosen as the independent variable. Nagelkerke's pseudo- $R^{2}$ statistics indicated that the regression models for the three dependent variables explained $5.5 \%$ (frequency), $13.8 \%$ (duration), and $6.8 \%$ (frequency $\times$ duration) of variance. In sum, for any rating level, higher affective priming effects predicted higher scores of reported frequency, duration, or overall amount of exercising (detailed statistics and comprehensive model 
specifications can be requested by the authors). Thus, the stronger the activation of positive targets relative to negative exercise-specific targets following exercise primes (i.e., the bigger the exercise-specific evaluative priming effect), the more the person exercises.

\section{Discussion}

The evaluative priming paradigm was used to show that exercisers differed significantly from nonexercisers when tapping into their impulsive systems. Priming effects show that exercisers responded faster to positive exercise-related target words after exercise primes. Nonexercisers responded faster to negative exercise-related target words after the same primes. These results indicate that exercisers hold positive associations toward exercise already on an automatic level. Nonexercisers hold less positive or even negative spontaneous associations. Aside from these differences in group means, ordinal regressions showed that evaluative priming effects predicted self-reported (structured questionnaire) frequencies of exercising, durations of typical exercise sessions, and overall amounts of exercising per week. These findings fit well into the broader picture that reflective processes may not solely account for the commitment to exercise.

In our study only exercise-specific positive and negative target words were able to produce the expected priming effects. The effect did not appear when generic (i.e., context-independent) positive and negative target words were used. This constitutes a difference from other priming research, where the hypothesized effects could be detected with generic targets words (Fazio et al., 1986). It is worthwhile to discuss this discrepancy in the light of other recent research.

To asses implicit attitudes toward walking in aircraftsmen trainees, Eves et al. (2007) also obtained some unexpected patterns of priming effects with Fazio's generic positive-negative categories: They report an overall negative priming effect. Active as well as inactive participants were quicker in responding to negative targets (thus suggesting that sport and physical activity are negatively evaluated by all subjects). Furthermore, and mirroring another aspect of our results, in their study the priming effect could only be demonstrated with domain-specific (there: mood-related) target stimuli.

First of all, what could have caused the discrepancy concerning the generic target task (overall-negative priming effect as reported by Eves et al. versus no priming effect in our study)? The simplest explanation is that different prime words were used. It is possible that the physical activity primes chosen by Eves et al. (e.g., squash, football, yoga, dancing) indeed represent a class of negatively evaluated physical activities. The authors themselves state that "the complexity of the pattern in these data dispels any simplistic notion that the positive and negative poles of affective priming may provide an implicit measure that maps easily onto behavior" (Eves et al., 2007, p. 582). We go along with such reservations. In our study, also, the expected group differences only emerge when nongeneric target words are used. And this is-second of all-exactly the point where Eves et al.'s and our results converge.

Unlike generic good-bad targets (e.g., appealing vs. repulsive in Fazio et al.'s studies or patient and corrupt in ours), both specific-target item pools (mood-related items, e.g., happy vs. miserable, in Eves et al.; exercise-specific items, e.g., athletic 
vs. exhausted in our study) consist of words that describe possible consequences of physical activity, exercise, or sport.

For example, after exercising one might feel athletic or exhausted (specific items). By contrast, one usually doesn't feel patient or corrupt after exercising (typical generic items). Generic items refer to rather abstract knowledge about a class of situations or persons; they may even represent stereotypes of people who themselves have not made live experiences with the primed concept. This reflects circumstances known from research on implicit attitudes: For example, you may be prejudiced toward a person although you have never met him or her. In the domain of exercise, both exercisers and nonexercisers can be shown to hold positive stereotypes toward the group of exercisers, regardless of their own activity status (Rodgers, Hall, Wilson, \& Berry, 2009). In contrast, the exercisespecific material may better address feelings associated with self-experienced behavior. The same argument may account for the stimuli Eves et al. (2007) have used. Accordingly, we argue that to assess automatic associations on behavior, experiential items are needed to render the evaluative priming task a valid measure and predictor of interindividual differences. This view should be able to initiate a more theory-driven discussion about the empirical finding (e.g., Gawronski \& Bodenhausen, 2006, 2007) that, in some studies, associations needed to be assessed context-dependently, whereas in others context-independent material showed to be sufficient.

If automatic processes play a role in exercise behavior, it is necessary to discuss their significance in the field of health behavior change. Most of the past research relied on behavior change models that exclusively represent aspects of the reflective system of human information processing. One example is TPB, where attitudes (together with perceived social norms and perceived behavioral control) determine the intention to behave. Integrative social cognition models such as RIM can lead to a better understanding of human behavior, because not only the reflective system, but also the impulsive system (i.e., automatic processes) are taken into account. Overcoming counteracting negative associations-and perhaps especially the ones that arise unintentionally and that tend to govern behavior in moments of fatigue and of relatively few mental control (Friese et al., 2008a, 2008b) - may be a substantial problem that has been widely neglected in traditional research on exercise and health behavior change so far.

As outlined in the opening paragraphs of this article, there is a line of research in which the relevance of the affective attitude component in exercise behavior has been demonstrated (Brand, 2006; Kiviniemi et al., 2007). These authors have used explicit measures, so that these evaluations should be assigned to processes characteristic of the reflective system. With the present research, we have demonstrated evaluative associations using an indirect measure. This provides evidence for the involvement of the impulsive system. It is worthwhile to consider the possibility that the affective attitude assessed by Brand (2006) and Kiviniemi et al. (2007) may be something like the rationalization of an underlying impulsive process.

Furthermore, the present work is capable of underscoring an idea brought forward by Mohiyeddini et al. (2009). These researchers have looked into an emotion-based approach for bridging the intention-behavior gap. While using self-report questionnaires, they have found that when the evaluative emotional appraisal of the intention is included as an additional variable in TPB, the prediction 
of behavioral variance could be improved substantially. According to Mohiyeddini et al., this is why researchers should start thinking about new intervention strategies, with which participants' evaluative appraisals about exercise intentions, and ultimately the behavior itself can be altered. In our eyes it is reasonable to widen this claim with regard to automatic evaluations.

As a concluding remark to these applied aspects of our discussion, it is important to stress that models highlighting the role of impulsive behavioral control in no way shall "compete" with well-founded intervention technology derived from evidence-based models of health behavior change that so far mainly addresses reflective processes. Due to their integrative nature, dual-system models such as RIM may complement these well-established perspectives instead.

Finally, there is a need to denote some study limitations, with which further research will have to deal. First, the route of causality needs to be clarified. Our study cannot answer the question of the origin of any existing differences. Regarding causality, we would expect that both influence directions are possible. Positive (negative) experiences with exercise may lead to positive (negative) associations, which subsequently instigate (inhibit) future engagement in exercising. But the reverse relation may also be true. Simply perceiving oneself to be relatively (in) active may induce positive (negative) associations toward exercising in line with principles of cognitive consistency, and alter the semantic network structures in the long run. Even though our study does not address the causal route of influence, we would maintain that —at the current stage of research-it is important to recognize that automatic evaluations of exercise do differ among participants, and that these differences can explain the amount of exercise to a sizable extent. An approach to gain insight into the question of causality could be to measure automatic evaluations of a sample of people before they start attending some kind of fitness or health intervention. Automatic associations as well as measures of intervention success (e.g., perseverance, dropout, extent of displayed exercise) can be recorded during the course of the physical activity-enhancing program in a longitudinal design, thereby allowing conclusions about causality to be drawn.

Second, incremental validity over deliberately reported evaluations (as it is usually done in social psychology attitude research) needs to be substantiated. Direct measures of evaluations connected with exercise behavior (e.g., questionnaires that separate cognitive from affective components of attitude) should be administered along with measures of automatic evaluations in a single study. It seems particularly fruitful to combine measures of automatic evaluations with objective measures of exercise, or, for example, with physiological outcomes of regular physical exercise. This approach could also remedy a shortcoming very common in research on evaluations (and attitudes), namely, that evaluative measures are used to predict only self-reported behaviors, but not (objectively observed) behavior itself.

Aside from these limitations, the most interesting challenge in future studies is to address our hypothesis that experiential targets are needed when behavior shall be explained in evaluative priming effects. Our concrete suggestion is to focus specifically on positive and negative consequences of exercise activities. Besides improving the method (i.e., using the evaluative priming task for predicting behavior), this could help to understand how affective evaluations are represented in the semantic network of the information processing system. 
Despite such limitations and open questions, we think the finding that evaluative priming effects are related to the degree of physical exercise contributes basically and essentially to the picture of reflective-plus-impulsive aspects of behavior control.

What might be considered as being good news at the theoretical level might be provoking at the level of intervention though. Up to now, little is known about the handling of possible (as we suppose: probable) counteractive interplays of reflective and impulsive behavioral tendencies in the course of interventions of health behavior change. Yet we are sure that as progress is made in understanding the underlying regularities of social information processing, applied exercise psychologists will be creative in designing corresponding intervention strategies.

\section{References}

Ajzen, I. (1991). The theory of planned behavior. Organizational Behavior and Human Decision Processes, 50, 179-211.

Baranowski, T., Anderson, C., \& Carmack, C. (1998). Mediating variable framework in physical activity interventions - How are we doing? How might we do better? American Journal of Preventive Medicine, 15, 266-297.

Baranowski, T., Lin, L.S., Wetter, D.W., Resnicow, K., \& Hearn, M.D. (1997). Theory as mediating variables: Why aren't community interventions working as desired? Annals of Epidemiology, 7, S89-S95.

Brand, R. (2006). Die affektive Einstellungskomponente und ihr Beitrag zur Erklärung von Sportpartizipation [The role of the affective attitudinal component in explaining physical exercise participation]. Zeitschrift für Sportpsychologie, 13, 147-155.

Chaiken, S., \& Trope, Y. (Eds.). (1999). Dual process theories in social psychology. New York: Guilford.

Degner, J., Wentura, D., \& Rothermund, K. (2006). Indirect assessment of attitudes with response-time-based measures: Chances and problems. Zeitschrift fur Sozial Psychologie, 37, 131-139.

Dunlap, W.P., Cortina, J.M., Vaslow, J.B., \& Burke, M.J. (1996). Meta-analysis of experiments with matched groups or repeated measures designs. Psychological Methods, $1,170-177$.

Evans, J.S.B.T. (2008). Dual-processing accounts of reasoning, judgment and social cognition. Annual Review of Psychology, 59, 255-278.

Eves, F.F., Scott, E.J., Hoppe, R., \& French, D.P. (2007). Using the affective priming paradigm to explore the attitudes underlying walking behaviour. British Journal of Health Psychology, 12, 571-585.

Fazio, R.H. (1990). Multiple processes by which attitudes guide behavior: The MODE model as an integrative framework. In M.P. Zanna (Ed.), Advances in Experimental Social Psychology (pp. 75-109). San Diego, CA: Academic Press.

Fazio, R.H., Jackson, J.R., Dunton, B.C., \& Williams, C.J. (1995). Variability in automatic activation as an unobstrusive measure of racial attitudes - A bona-fide pipeline. Journal of Personality and Social Psychology, 69, 1013-1027.

Fazio, R.H., \& Olson, M.A. (2003). Implicit measures in social cognition research: Their meaning and use. Annual Review of Psychology, 54, 297-327.

Fazio, R.H., Sanbonmatsu, D.M., Powell, M.C., \& Kardes, F.R. (1986). On the automatic activation of attitudes. Journal of Personality and Social Psychology, 50, 229-238.

Friese, M., Hofmann, W., \& Schmitt, M. (2008a). When and why do implicit measures predict behaviour? Empirical evidence for the moderating role of opportunity, motivation and process reliance. European Review of Social Psychology, 19, 285-338. 
Friese, M., Hofmann, W., \& Wänke, M. (2008b). When impulses take over: Moderated predictive validity of explicit and implicit attitude measures in predicting food choice and consumption behaviour. The British Journal of Social Psychology, 47, 397-419.

Gawronski, B., \& Bodenhausen, G.V. (2006). Associative and propositional processes in evaluation: An integrative review of implicit and explicit attitude change. Psychological Bulletin, 132, 692-731.

Gawronski, B., \& Bodenhausen, G.V. (2007). Unraveling the processes underlying evaluation: Attitudes from the perspective of the ape model. Social Cognition, 25, 687-717.

Hagger, M.S., Chatzisarantis, N.L.D., \& Biddle, S.J.H. (2002). A meta-analytic review of the theories of reasoned action and planned behavior in physical activity: Predictive validity and the contribution of additional variables. Journal of Sport \& Exercise Psychology, 24, 3-32.

Haskell, W.L., Lee, I.M., Pate, R.R., Powell, K.E., Blair, S.N., Franklin, B.A., et al. (2007). Physical activity and public health : Updated recommendation for adults from the American college of sports medicine and the American heart association. Circulation, 116, 1081-1093.

Hofmann, W., Friese, M., \& Wiers, R.W. (2009). Impulsive versus reflective influences on health behavior: A theoretical framework and empirical review. Health Psychology Review, 1, 1-27.

Kesaniemi, Y.A., Danforth, E., Jensen, M.D., Kopelman, P.G., Lefebvre, P., \& Reeder, B.A. (2001). Dose-response issues concerning physical activity and health: An evidence based symposium. Medicine and Science in Sports and Exercise, 33(Suppl. 6), 531-538.

King, A.C. (2001). The coming of age of behavioral research in physical activity. Annals of Behavioral Medicine, 23, 227-228.

Kiviniemi, M.T., Voss-Humke, A.M., \& Seifert, A.L. (2007). How do I feel about the behavior? The interplay of affective associations with behaviors and cognitive beliefs as influences on physical activity behavior. Health Psychology, 26, 152-158.

Lieberman, M.D. (2007). The X-and C-systems: The neural basis of automatic and controlled social cognition. In E. Harmon-Jones \& P. Winkielman (Eds.), Fundamentals of Social Neuroscience (pp. 290-315). New York: Guilford.

Lippke, S., Ziegelmann, J.P., \& Schwarzer, R. (2004). Behavioral intentions and action plans promote physical exercise: A longitudinal study with orthopedic rehabilitation patients. Journal of Sport \& Exercise Psychology, 26, 470-483.

Marshall, S.J., \& Biddle, S.J.H. (2001). The transtheoretical model of behavior change: A meta-analysis of applications to physical activity and exercise. Annals of Behavioral Medicine, 23, 229-246.

McAuley, E., Jerome, G.J., Elavsky, S., Marquez, D.X., \& Ramsey, S.N. (2003). Predicting long-term maintenance of physical activity in older adults. Preventive Medicine, $37,110-118$.

Mohiyeddini, C., Pauli, G., \& Bauer, S. (2009). The role of emotion in bridging the intention- behaviour gap: The case of sports participation. Psychology of Sport and Exercise, 10, 226-234.

Prochaska, J.O., \& DiClemente, C.C. (1983). Stages and processes of self-change of smoking- Toward an integrative model of change. Journal of Consulting and Clinical Psychology, 51, 390-395.

Prochaska, J.O., Velicer, W.F., Rossi, J.S., Goldstein, M.G., Marcus, B.H., Rakowski, W., et al. (1994). Stages of change and decisional balance for 12 problem behaviors. Health Psychology, 13, 39-46.

Rodgers, W.M., Hall, C.R., Wilson, P.M., \& Berry, T.M. (2009). Do nonexercisers also share the positive exerciser stereotype?: An elicitation and comparison of beliefs about exercisers. Journal of Sport \& Exercise Psychology, 31, 3-17.

Rydell, R.J., \& Gawronski, B. (2008). I like you, I like you not: Understanding the formation of context-dependent automatic attitudes. Cognition and Emotion, 23, 1118-1152. 
Sallis, J.F. (2001). Progress in behavioral research on physical activity. Annals of Behavioral Medicine, 23, 77-78.

Scholz, U., Sniehotta, F.F., \& Schwarzer, R. (2005). Predicting physical exercise in cardiac rehabilitation: The role of phase-specific self-efficacy beliefs. Journal of Sport \& Exercise Psychology, 27, 135-151.4

Schwarz, N., \& Oyserman, D. (2001). Asking questions about behavior: Cognition, communication, and questionnaire construction. The American Journal of Evaluation, 22, $127-160$.

Schwarzer, R. (1992). Self-efficacy in the adoption and maintenance of health behaviors: Theoretical approaches and a new model. In R. Schwarzer (Ed.), Self-efficacy: Thought Control of Action (pp. 217-243). Washington, DC: Hemisphere.

Schwarzer, R. (2008). Modeling health behavior change: How to predict and modify the adoption and maintenance of health behaviors. Applied Psychology: An International Review, 57, 1-29.

Sheeran, P. (2002). Intention-behavior relations: A conceptual and empirical review. European Review of Social Psychology, 12, 1-36.

Sjöström, M., Oja, P., Hagströmer, M., Smith, B.J., \& Bauman, A. (2006). Health-enhancing physical activity across european union countries: The Eurobarometer study. Journal of Public Health, 14, 291-300.

Smith, E.R., \& DeCoster, J. (2000). Dual-process models in social and cognitive psychology: Conceptual integration and links to underlying memory systems. Personality and Social Psychology Review, 4, 108-131.

Strack, F., \& Deutsch, R. (2004). Reflective and impulsive determinants of social behavior. Personality and Social Psychology Review, 8, 220-247.

Webb, T.L., \& Sheeran, P. (2006). Does changing behavioral intentions engender behaviour change? A meta-analysis of the experimental evidence. Psychological Bulletin, 132, 249-268.

Zanna, M.P., \& Rempel, J.K. (1988). Attitudes: A new look at an old concept. In D. Bar-Tal \& A.W. Kruglanski (Eds.), The Social Psychology of Knowledge (pp. 315-334). New York: Cambridge University Press.

Manuscript received: January 15, 2009

Revision accepted: October 4, 2009 